\title{
White Label et " péril jaune » : race, genre et travail en Californie, fin XIXe-début XXe siècle
}

\section{Nayan SHAH}

\section{(2) OpenEdition \\ 1 Journals}

Édition électronique

URL : http://journals.openedition.org/clio/464

DOI : 10.4000/clio.464

ISSN : $1777-5299$

Éditeur

Belin

Édition imprimée

Date de publication : 1 avril 1996

ISSN : 1252-7017

Référence électronique

Nayan SHAH, « White Label et « péril jaune » : race, genre et travail en Californie, fin XIXe-début XXe siècle », Clio. Histoire, femmes et sociétés [En ligne], 3| 1996, mis en ligne le 01 janvier 2005, consulté le 20 avril 2019. URL : http://journals.openedition.org/clio/464 ; DOI : 10.4000/clio.464

Ce document a été généré automatiquement le 20 avril 2019.

Tous droits réservés 


\title{
White Label et " péril jaune » : race, genre et travail en Californie, fin XIXe-début XXe siècle
}

\author{
Nayan SHAH
}

La présence de Chinois qui ont afflué en nombre excessif [à San Francisco] est dangereuse pour la santé des habitants en raison du surpeuplement des logements de ces Chinois, des maladies qu'ils introduisent et de la crasse extrême et invétérée de leurs lieux de résidence. ${ }^{1}$

Chinatown est une invitation permanente au choléra, à la variole et autres pestes... Tout le quartier où réside cette race étrangère est infesté par la putréfaction; la puanteur qui s'échappe de leurs tanières en sous-sol et se répand sur toute la ville est chargée de germes mortels pour une race différente et supérieure. ${ }^{2}$

Pour beaucoup d'Américains blancs, l'importante immigration de travailleurs chinois en Californie au XIXe siècle représentait une menace aux multiples visage; menace qui mettait en danger leur travail, leur santé, leur mode de vie - en un mot, tout le " progrès » qu'ils avaient accompli en tant que citoyens et ouvriers. Comme le suggèrent la déclaration, citée ci-dessus, du San Francisco Council au début de l'immigration chinoise et les remarques du Docteur Mary Sawtelle 24 ans plus tard, la représentation mentale collective du péril que feraient courir les Chinois à la santé publique, était un élément déterminant dans l'élaboration de cette prétendue menace pour les familles américaines et leurs moyens d'existence ${ }^{3}$. Les syndicats ouvriers américains se sont emparés de cette image collective et l'ont utilisée comme arme dans la campagne organisée en 1875 par la Cigar Maker's Association [Association des ouvriers cigariers], exclusivement blanche, de la côte Pacifique. Cette année-là les ouvriers cigariers commencèrent à marquer leurs produits avec un label blanc qui faisait office de déclaration raciale informant explicitement les consommateurs éventuels que les cigares avaient été fabriqués par des « hommes blancs ». Encouragés par ce discours bien structuré sur le danger médical - qui circulait alors largement parmi les médecins, les officiels de la santé publique et les politiciens, aussi bien que chez les militants syndicaux - le syndicat en appelait directement aux consommateurs blancs pour qu'ils créent « une demande de cigares fabriqués par les Blancs et, plus généralement, pour qu'ils obligent les fabricants à 
employer uniquement une main d'œuvre blanche $»^{4}$. A travers une analyse des discours sur le danger racial, la consommation hygiénique et la répartition sexuée des rôles qui préserve la sécurité de la famille, cet article étudie comment un langage racialement codé sur la santé et l'hygiène commença à s'insérer dans les combats économiques et politiques du mouvement ouvrier du début du XXe siècle.

Critères raciaux et société hygiéniste

En Californie, la consolidation du mouvement ouvrier a été fondée sur un harcèlement racial et une violence haineuse à l'encontre des Chinois et le "péril jaune » sur la santé publique a fourni un alibi puissant et supposé scientifique pour une politique du travail anti-chinoise $\mathrm{e}^{5}$. Le premier white label inventé par l'Association des ouvriers cigariers a nettement favorisé la formation naissante de l'identité banche qui avait défini l'appartenance au syndicat sur l'exclusion et donné naissance à la solidarité raciale pour atténuer des conflits éventuels entre les nationalités européennes de l'immigration et les groupes religieux ${ }^{6}$. Au XIXe siècle les syndicalistes ouvriers en accusant les ouvriers chinois cigariers de déposer des " germes infectieux » de lèpre et de syphilis en roulant le tabac et en humidifiant le bout des cigares attira l'attention et l'intervention régulatrice des autorités de la santé publique ${ }^{7}$. Ces accusations furent développées avec plus de force dans le cadre de l'enquête du Workingman's Party of California (WPC) [Parti des travailleurs de Californie] sur les conditions de santé publique à «Chinatown » en 1880, qui fut effectuée en association avec une bienveillante enquête officielle du Ministère de la santé. Le WPC concluait :

Des entreprises [chinoises] de différentes sortes - de cigares, de vêtements, etc. entreposent même des produits alimentaires dans les trous les plus crasseux que l'on puisse imaginer - soumises dans de telles antres à l'imprégnation de germes infectieux qui doivent être préjudiciables à la santé du consommateur ou de l'usager... La propagation de ces germes porteurs de maladie et leur introduction au sein des familles sont, outre cette infection venant des produits, grandement favorisées par les blanchisseries chinoises et les serviteurs chinois de ces familles... un réseau parfait de contagion et d'infection est ainsi créé, une véritable pieuvre de maladie ayant son siège à Chinatown et dont les bras infectieux se frayent un passage jusqu'à l'intérieur de chaque foyer de la ville. ${ }^{8}$

4 L'hystérie sur la propagation de maladies raciales masquait cependant les dramatiques transformations structurelles qui avaient rendu vulnérables économiquement les artisans qualifiés de l'industrie du cigare et d'autres ouvriers blancs. Les changements dans les processus de production rendaient les compétences des artisans cigariers de plus en plus obsolètes dans la mesure où les employeurs tiraient plus de profit de l'embauche d'ouvriers immigrés chinois, européens ou latino-américains moins qualifiés9. Le combat pour le maintien d'une production artisanale qualifiée conduisit le Cigar Maker's International Union (CMIU) [Syndicat international des ouvriers cigariers] à adopter la pratique du label syndical en 1880. Le CMIU avait adapté le libellé du label aux questions concernant le travail qualifié, l'organisation syndicale et les conditions sanitaires et avait incorporé celles-ci dans les différences structurelles des marchés de la production et du travail. Leur label bleu clair avec une garantie de «fabrication soignée de première classe » fut acclamé comme un «bon détective permettant de séparer et d'éliminer la fabrication réalisée dans les immeubles crasseux de second ordre, dans les prisons, les habitations des Chinois et les échoppes à rats » qu'on avait frauduleusement mis sur le même pied que la production «mécanique qualifiée $»^{10}$. Le débat explicite sur l'identité raciale blanche fut remplacé par des marques rhétoriques d'infériorité raciale - les 
désignations interchangeables de «Chinois» et «coolie $»^{11}$ - et par une panoplie de pratiques d'emploi «injustes» qui menaçait l'organisation syndicale, en l'occurrence l'embauche de prisonniers, de nouveaux immigrants européens et d'ouvriers noirs ${ }^{12}$.

Pendant pratiquement les vingt années qui ont précédé la Première Guerre mondiale, les significations raciales de la solidarité ouvrière furent élaborées à l'occasion des campagnes à l'intention de consommateurs menées par le San Francisco Labor Council [Conseil du travail de San Francisco]. Son hebdomadaire officiel, le Labor Clarion [Le clairon du travailleur] présentait périodiquement des rapports organisationnels et des mémoires convaincants qui développaient le programme conceptuel et pratique de la campagne « Achetez le label syndical ». Formule syndicaliste typiquement américaine, le label syndical bénéficia d'une popularité croissante aux États-Unis dans les années 1890 et fut rapidement adopté par des dizaines de syndicats nationaux. En 1908 soixante-huit des cent soixante-dix syndicats nationaux affiliés à l'American Federation of Labor (AFL) [Fédération américaine du travail] utilisaient ce label. Les syndicats utilisant ce label totalisaient un nombre de 724.000 adhérents, ce qui représentait $47 \%$ de l'ensemble des adhérents de l'AFL. Au début du siècle les syndicats utilisèrent de plus en plus ce label à l'échelle internationale en Angleterre, en Australie et en France ${ }^{13}$.

6 Après des procès répétés devant les tribunaux à travers les États-Unis, les inscriptions raciales explicites furent supprimées des labels, cependant l'exclusion raciale fut maintenue dans l'organisation et la rhétorique des campagnes destinées aux consommateurs. La propagande en faveur du label syndical soulignait avec fierté les origines anti-chinoises de ce label et, de plus, elle applaudissait aux pratiques toujours en vigueur qui excluaient de l'économie les Asiatiques et autres "inassimilables ${ }^{14}$. La consommation de produits fabriqués sous contrôle syndical était un devoir patriotique américain dans l'intérêt du travail qualifié. L'adhésion au label syndical était considérée comme cruciale à cette époque d'intense immigration européenne et asiatique que les dirigeants syndicalistes décriaient parce qu'elle avait apporté une " horde d'ouvriers bon marché et non qualifiés » sur les rivages américains. Le "modèle de vie » américain ne pouvait être maintenu que par une stricte distinction entre l'immigrant assimilable qui était venu pour « être parmi nous, partager nos libertés, adopter nos coutumes et nos lois, avancer et progresser avec nous » et le " coolie chinois, la boutique suante et les classes colonisées qui représentaient un mode de vie bien trop bas pour être adoptés par nos concitoyens $»^{15}$. Le dirigeant syndical du Massachusetts, John Graham Brooks prétendait que la nécessité de préserver un modèle de vie 'américain' l'emportait sur les idéaux ouvriers universels de fraternité et d'égalité politique. Pour Brooks, le choix dans la consommation des cigares devenait une décision permettant de «maintenir le modèle de vie et de travail des Blancs par opposition à celui des coolies $»^{16}$.

Dans la politique américaine du travail au début du siècle, la rhétorique du modèle de vie se caractérisait par des codes interchangeables de race et de nationalité; le soi-disant modèle de vie 'américain' ou plus carrément le «modèle de vie du Blanc» se définissait par une opposition radicale avec celui de "l'Asiatique » ou du «coolie $»^{17}$. L'AFL, fondée en 1881, s'engagea avec persévérance dans une croisade politique visant à interdire aux Chinois et aux autres ouvriers asiatiques de rejoindre les rangs du syndicat et de la nation. Samuel Gompers ${ }^{18}$, qui fut longtemps président de l'AFL et Herman Gutstadt, permanent de la Cigar Maker's Union, installé à San Francisco, signèrent ensemble un essai qui fut diffusé nationalement et qui rassemblait cinquante années de rhétorique anti-chinoise dans une explication de la façon dont les modèles de vie des Chinois étaient 
incompatibles avec l'édifice politique et culturel de la société américaine et même destructeurs à son égard ${ }^{19}$. « On ne peut faire travailler un homme qui a besoin de bœuf et de pain à côté d'un homme qui vit de riz. Dans tous les conflits et tous les combats de ce genre, le résultat n'est pas de hisser l'homme qui vit de riz au niveau du modèle bœuf-etpain mais de rabaisser l'homme vivant de bœuf et de pain au modèle du riz $»^{20}$. Ils prédisaient qu'une nette différence entre les salaires des Chinois qui étaient censés être des mangeurs de riz et les Blancs mangeurs de bœuf entraînerait l'effondrement du niveau de salaires de ces derniers, ce qui diminuerait leur confiance en l'ordre politique et susciterait ainsi le mécontentement et la sédition.

Dans l'espoir de garantir la reconduction de la législation restreignant l'immigration chinoise en 1902, Compers et Gutstadt firent appel à la rhétorique des modèles de vie selon les races, de la stabilité nationale et de l'instinct de conservation masculine en vogue au moment de la politique anti-chinoise des années 1870. Les schémas ouvriers contrastés de consommation alimentaire étaient des codes abrégés qui nourrissaient les inquiétudes sur la baisse de la mobilité sociale. Durant le XIXe siècle, l'assaut structurel contre la production artisanale mit les ouvriers qualifiés dans l'impossibilité croissante de prendre en charge, par leur seul travail, femme et enfants - élément clé de l'idéal viril de la classe ouvrière. Pour les ouvriers l'affirmation d'une masculinité indépendante et respectable se structurait autour de leur capacité à avoir des personnes à charge et de les entretenir ${ }^{21}$. Les ouvriers chinois devinrent des boucs émissaires lorsque les conditions structurelles - salaires en baisse, déqualification, chômage cyclique, embauche des femmes et des enfants pour le travail en usine - rendirent cet idéal impossible à atteindre. Puisque les Chinois étaient considérés comme des célibataires sans obligations familiales, le modèle « coolie » fut également investi des peurs d'un monde socialement désorganisé, constitué d'hommes sans emploi, de femmes prostituées et de familles ouvrières déchirées par le vice, la pauvreté et la maladie.

Cet imaginaire horrible d'un monde de vices, de maladies, de pauvreté, de prostitution, où n'existait aucune véritable famille, aucun véritable foyer reflétait déjà les caricatures existantes des conditions de vie des Chinois qui étaient elles-mêmes considérées comme le résultat direct des bas salaires, des longues heures de travail et des conditions insalubres de ce travail. La contradiction que cela représentait avec la vision progressiste de ce que devait être le monde ouvrier blanc était intentionnelle. Elle servait à renforcer la signification de sa contre-image: les récents mouvements couronnés de succès en faveur de conditions de travail salubres et sans danger dans les usines, les croisades pour des logements spacieux et des espaces verts dans la ville ainsi que les campagnes pour obtenir des journées de travail de huit heures et pour bannir le travail des enfants. Dans la mesure où ces réformes étaient incertaines et inégales, les activistes ouvriers étaient extrêmement sensibles à toute menace visible contre les avantages durement acquis et utilisaient l'occasion pour affirmer que ces nouvelles conditions devaient être la norme et qu'elles étaient nécessaires à la préservation de l'ordre social et de la nation. Ainsi, la caricature du «modèle oriental » avait pour fonction de consolider le soutien moral et l'influence politique du programme syndical destiné à fixer les règles du monde industriel. La régulation apparaissait comme cruciale pour garantir une vie humaine aux familles ouvrières, pour préserver la race blanche et pour maintenir la stabilité communautaire.

10 Les références à l'hygiène dans le label syndical étaient devenues de plus en plus significatives dans les définitions des modèles de vie en raison de la force de la réforme 
sur la santé publique dans les initiatives syndicales. La réforme sociale et les campagnes ouvrières du début du siècle pour éradiquer les taudis, améliorer l'hygiène, rendre les usines responsables face aux normes d'hygiène industrielle et obliger le monde de l'industrie à se débarrasser des pratiques du travail dans les ateliers et à domicile servaient de prémices à la protection de la santé et de la sécurité des ouvriers du syndicat et de leurs familles ${ }^{22}$. Dans les quartiers ouvriers urbains comme dans les usines, le plaidoyer en faveur de "conditions salubres » sur le lieu de travail et à la maison avait obtenu une large approbation politique car il s'agissait de projets de réforme et de régulation raisonnables et humains. Les promoteurs du label déclaraient que les marchandises qui n'en étaient pas munies étaient «fabriquées dans des ateliers, des prisons et des sous-sols mal aérés, surpeuplés, d'où les maladies se propageaient, par des esclaves surchargés de travail et mal payés par des employeurs cupides ", mais aussi que l'achat des produits bénéficiant du label appuyait la croisade en faveur de conditions de travail salubres dans les usines ${ }^{23}$.

11 Les partisans du label syndical mirent au premier plan l'association entre l'organisation syndicale et le plaidoyer en faveur de conditions de travail salubres. Ils encouragèrent la consommation des produits fabriqués par le syndicat car ils représentaient une " garantie » essentielle contre « l'atelier infâme, engendrant des maladies » qui déversait « les germes de maladie et de mort aux quatre coins du pays tant que leurs produits pouvaient être vendus». Ils essayèrent d'établir un lien entre la production et la consommation en déclarant que "des conditions [de travail] insalubres pour l'ouvrier... signifient douleur, maladie et mort pour le consommateur $»^{24}$. En «garantissant» le consommateur contre la " crasse et la contagion », le label syndical apparaissait comme le moyen d'organiser « le pouvoir d'achat en fonction de justes conditions de travail et aussi contre ces conditions qui détruisent la santé et la moralité du producteur et qui mettent en danger le bien être de l'acheteur $»^{25}$. La plus grande partie de la publicité commerciale directe au sujet de la « garantie » du label syndical jouait sur les peurs de propagation des maladies. Dans une série d'encarts publicitaires de l'United Garment Workers [Ouvriers de la confection unis] la légende accompagnant les costumes pour hommes informait le lecteur que «les vêtements portant ce label garantissent l'acheteur contre la contagion. Assurent que le vêtement vient d'une boutique moderne et propre. Qu'il a été fabriqué par des tailleurs syndiqués qualifiés $\aleph^{26}$. Le syndicat des ouvriers cigariers entama une nouvelle et énergique campagne de publicité et d'agitation sur le label qui présentait leurs produits comme des «ennemis de la saleté et de la maladie dont les cigares fabriqués par les Chinois et dans les immeubles d'habitation sont des exemples ». Certains dirigeants du syndicat allèrent même jusqu'à rechercher l'appui de médecins certifiant que les cigares fabriqués au sein du syndicat étaient produits de façon hygiénique et que de ce fait « ils évitaient certainement la transmission de germes ${ }^{27}$.

12 Le sens donné à la «salubrité » des lieux de travail reposait souvent sur les visions de la fin du XIXe siècle de la séparation des endroits de vie et de travail. Le mélange « immoral » du loisir et du travail avait, durant les décennies précédentes, alarmé les autorités en charge de la santé publique qui craignaient que le mal et l'immoralité "contaminent» les produits de consommation. On faisait état des possibilités "d'infection» par négligence sur une illustration intitulée "Scène dans une usine chinoise de balais » qui accompagnait un article où le travail des prisonniers et celui des Chinois étaient assimilés aux deux menaces jumelles pesant sur le syndicat blanc des ouvriers de la fabrication de balais ${ }^{28}$. Le dessin mettait en lumière le mélange douteux 
d'activités - manger, fumer de l'opium et dormir - dans un atelier désordonné et bondé où seuls quelques hommes fabriquaient réellement des balais. L'image rassemblait les représentations typiques du vice et du désordre des Chinois avec des insinuations plus générales sur la paresse et la faible productivité qui n'étaient habituellement pas attribuées aux ouvriers chinois. La "racialisation» de l'usine de balais prenait de l'importance à partir de l'utilisation d'images de «surpeuplement », de fumerie d'opium et de l'amalgame général du travail et des loisirs qui avait exaspéré les inspecteurs de la santé de San Francisco en charge des blanchisseries et des manufactures de cigares à la fin du XIXe siècle et qui avait déjà été accusé de transmettre des maladies aux consommateurs ${ }^{29}$.

Appels sexués et défense du foyer

14 La focalisation du label syndical sur la consommation poussa à une nouvelle articulation du rôle des deux sexes dans le discours ouvrier. L'AFL, le Clarion et d'autres institutions syndicales se montraient désireuses de développer une rhétorique qui encouragerait les femmes à participer au mouvement en faveur du label syndical. En 1904, l'AFL finança un concours de textes sur ce sujet. Le texte primé, écrit par Walter MacArthur éditeur du Coast Seaman's Report [Bulletin du marin de la côte] de San Francisco, formulait une constellation de valeurs et de devoirs, clairement sexués, des hommes et des femmes dans la promotion du label syndical. Pour les hommes, «le label syndical signifie l'application à la vie industrielle de ces règles que chaque bon citoyen applique dans sa vie personnelle - propreté, moralité, honnêteté, courtoisie envers les femmes et prise en charge des plus jeunes ». Pour les femmes la signification était plus complexe: MacArthur croyait que «les instincts des femmes et les intérêts ouvriers se rejoignaient dans le label syndical. Les deux défendent la propreté, la moralité, la prise en charge des enfants [et] le caractère sacré du foyer ». En consommant des produits fabriqués sous contrôle syndical, la «maîtresse de maison » peut exercer son pouvoir d'achat en faveur du syndicat : «En exigeant le label syndical la femme du syndicaliste devient vraiment la compagne (the helpmeet) [sic] du soutien de famille (the breadwinner), sa puissante influence s'étendant ainsi du foyer à l'atelier duquel elle serait autrement totalement exclue $»^{30}$.

Les femmes des familles syndicalistes organisèrent la Woman's Union Label League [Ligue des femmes pour le label syndical] qui se ramifia à travers les États-Unis et le Canada, avec en particulier une organisation active à San Francisco ${ }^{31}$. Beaucoup de femmes favorables au label syndical cherchaient à élargir, dans le combat politique du syndicat, le rôle des ménagères de la classe ouvrière. En 1905, Mme Mamie Brettel, présidente et organisatrice générale de la Woman's International Union Label League (WIULL) déclarait que l'organisation existait pour «enseigner aux femmes comment travailler ensemble pour l'amélioration de l'humanité, travail que chacune commence dans son foyer, avec son travail quotidien, mais qui ne se termine que par la victoire finale sur le mal $»^{32}$. L'année suivante Annie Fitzgerald, alors présidente de la WIULL, affirmait que l'éducation des femmes sur le label syndical devait prendre en compte leur pouvoir en tant " qu'acheteuses » puis guider ce pouvoir grâce à une compréhension éclairée des intérêts politiques et économiques : si une femme syndiquée « achète un vêtement ne portant pas le label syndical pour elle ou pour son enfant, elle travaille contre les véritables intérêts de son mari dont le métier peut être représenté par un label syndical $»^{33}$.

On fait constamment référence aux femmes dans leur rôle de garant du « foyer » et de la «famille»- «gardiennes du revenu familial » et «préposées aux achats du foyer ». Les femmes étaient les épouses, les sœurs, les mères ou les filles des hommes du syndicat. Les 
suppositions de MacArthur et Fitzgerald sur la division stricte et sexuée des rôles dans la classe ouvrière étaient largement partagées par leurs collègues syndicalistes. Les représentations de la vie domestique des femmes de la classe ouvrière singeaient les rôles et les sphères séparées de la classe moyenne et niaient l'existence des femmes comme ouvrières dans les usines, les ateliers ou à domicile ${ }^{34}$. Ces rôles sexués « convenables » qui assignaient les femmes à la sphère du domestique, du travail non payé, cadraient aussi fort bien avec les programmes de réformes du travail et de la santé publique qui cherchaient à fermer les sweatshops et à réduire le travail industriel à domicile, ce qui revenait effectivement à éloigner beaucoup de femmes et d'enfants du marché du travail.

En formulant une nouvelle articulation des rôles domestiques des femmes, les promoteurs du label syndical cherchaient à enseigner aux femmes de la classe ouvrière les tactiques et les impératifs du monde du travail en dépit de leur "indifférence " supposée au syndicalisme. Cette condescendance démentait la politique de plusieurs syndicats de l'AFL consistant à ouvrir leurs rangs aux femmes et l'empressement de nombreuses femmes à adhérer ${ }^{35}$. Afin de maintenir la différenciation des genres dans le sillage des transformations organisationnelles, les dirigeants syndicalistes firent appel aux femmes en tant que figures domestiques et mirent l'accent sur les améliorations apportées dans le foyer et la famille grâce à une consommation consciente des femmes: de meilleurs salaires et moins d'heures de travail pour le "soutien de famille" se traduirait par un "confort " accru dans le ménage, ainsi que des améliorations dans la santé familiale et moins de travail pour les femmes au foyer. Cette politique syndicale déniait le travail extérieur des femmes et renforçait leur dépendance envers les ouvriers mâles ${ }^{36}$.

Les syndicats membres du San Francisco Labor Council et la section locale de la Women's Union Label League développèrent des programmes publicitaires pour atteindre les consommatrices. En octobre 1905 le Label Committee du Labor Council fit circuler 50.000 tracts adressés à « la maîtresse du ménage » dans tous les foyers de la ville. Ces tracts appelaient les femmes à aider leurs maris, pères et frères à obtenir des salaires corrects, un nombre raisonnable d'heures de travail, à réduire la nécessité des grèves et à favoriser les chances de succès des lockouts en achetant les produits portant le label syndical ${ }^{37}$. L'année suivante, le Council distribua dans 50.000 foyers de la ville un calendrier du label syndical qui valorisait les labels, timbres, cartes de magasins et de boutiques du syndicat. Ces calendriers sont devenus une tradition populaire. Dans les années qui suivirent, le Labor Council finança la distribution de cartes postales illustrées portant des slogans en faveur du label et la production d'un spectacle de music-hall qui vantait les mérites de la consommation des produits labélisés par l'intermédiaire de spectacles de variétés et de discours publics ${ }^{38}$.

19 L'importance grandissante des femmes pour mener à bien les campagnes syndicales sur l'organisation et la consommation ainsi que l'accroissement notable des dirigeantes dans les syndicats, les ligues en faveur du label et les associations de quartier incitèrent le Clarion à consacrer une page aux problèmes des femmes et aux informations les concernant. Mme E.H. Donnerl, imprimeuse, syndicaliste et militante de quartier, faisait office de rédactrice en chef du Women's Deparment [Département femmes], chronique régulière du Clarion d'août 1908 à 1911. Sous la houlette de Mme Donnel, le Women's Department publiait des informations sur les syndicats et les ligues en faveur du label, des articles concernant le travail des femmes et des enfants, et des conseils sur la tenue de la maison et des recettes de cuisine ${ }^{39}$. La notion d'un département ou d'une colonne 
spécifique «femmes" renforçait l'idée de problèmes politiques et sociaux séparés du monde présumé masculin du reste du Clarion. Cela fonctionnait aussi comme une concession du syndicat ouvrier dominé par les hommes envers la citoyenneté particulière des femmes blanches.

Cette citoyenneté particulière se distinguait par des modes de dépendance. Les questions soulevées, spécifiques "aux femmes", correspondaient à un programme syndical global. Par exemple, la question du travail des enfants était perçue comme un lien entre les syndicats et les organisations humanitaires des femmes des classes moyennes. On considérait le travail des enfants en fonction des conséquences particulièrement affreuses pour le foyer, la famille et la nation. Les femmes étaient recrutées pour défendre l'adoption de lois destinées à "aider à enlever l'enfant d'âge tendre de l'usine et à le mettre dans des écoles et des cours de récréation, là où il devrait être; cela l'aidera à devenir un homme sain, et donc en fera un bon citoyen $»^{40}$. Dans le discours, la mise hors la loi du travail des enfants satisfaisait au programme du mouvement national naissant sur la santé maternelle et la protection de l'enfance. Elle a de fait éliminé la main d'œuvre enfantine lorsque celle-ci sapait financièrement le travail syndiqué, mais elle a perturbé la vie des enfants et rendu leurs familles beaucoup plus vulnérables économiquement. Malgré ces difficultés, les femmes étaient censées trouver une satisfaction dans le retrait des garçons du marché du travail et dans leur admission à l'école qui leur permettraient de se réaliser pleinement à l'âge d'homme.

21 Les femmes syndicalistes qui écrivaient dans le journal ne se concentraient pas toutes sur l'élargissement de la citoyenneté aux ouvriers, hommes et jeunes garçons. Certaines dirigeantes commencèrent à réévaluer le contenu de leur propre citoyenneté et leur capacité à fabriquer plutôt qu'à influencer la législation. En 1909, la page consacrée aux femmes vit une augmentation régulière des reportages destinés à promouvoir le droit de vote pour les femmes ${ }^{41}$, en reproduisant les discours et les commentaires qui reliaient les débats féministes progressistes sur la nécessité de la citoyenneté par le vote pour prendre en charge la gestion municipale aux intérêts des syndicalistes pour la garantie de la souveraineté du syndicat dans les usines et la salubrité du lieu de travail. En 1912, après le vote en Californie de l'amendement en faveur du droit de vote des femmes, le San Francisco Labor Council finança la mobilisation électorale des « ouvrières et des épouses, filles et parentes féminines des ouvriers ». Peu après, la Women's Humane Legislation League [Ligue pour une législation humaine pour les femmes] fut créée par le Labor Council, le Building Trades Council et la Women's Union Label League afin d'organiser les femmes de la classe ouvrière en groupe de pression votant en faveur du syndicat. La militante Alice Park proposa un "programme des femmes» qui soutenait les lois instaurant des journées de travail de huit heures pour les femmes et les enfants et défendait les causes féministes y compris la limitation du "quartier réservé » (red light district), l'égalité des salaires et la réforme des lois sur la propriété et la garde des enfants afin que les femmes acquièrent un plus grand contrôle sur les affaires financières et sur leur progéniture ${ }^{42}$.

22 L'arène politisée des syndicats devint, pour les femmes de la classe ouvrière, un forum leur permettant de former des coalitions fondées sur les ressemblances de genre qui transcenderaient les différences de classes. La direction masculine du syndicat voyaient dans ces coalitions féminines interclassistes un moyen d'élargir le soutien au programme syndical ; les femmes réformistes et suffragettes des classes moyennes y voyaient, elles, la possibilité d'entraîner les femmes et les hommes de la classe ouvrière à soutenir leur 
programme en faveur de leur politique de moralisation, de l'indépendance économique et sociale des femmes et de la sécurité sociale. Grâce à la politisation des femmes blanches de la classe ouvrière, cette coalition se déployait d'une part contre les riches industriels et d'autre part contre les ouvriers privés de droit de représentation et non syndiqués (les minorités raciales, les récents immigrants européens et les prisonniers). Cette alliance devrait être comprise comme une réponse aux inquiétudes raciales qui hantaient les Blancs, hommes et femmes. Leur intérêt pour l'amélioration des conditions de travail et du milieu social des syndicalistes (blancs) s'accordait avec leur désir de maintenir les hiérarchies et les exclusions raciales dans l'ordre social et le système économique.

Race, santé et travail

Les dirigeants syndicaux supposaient qu'il existait un lien entre blancs et santé, Asiatiques et maladie, en utilisant pour preuves des statistiques et des anecdotes tirées de certaines strates du savoir vulgarisé sur la santé publique. Dans les premières décennies du XXe siècle, des campagnes d'éducation publique, des organisations gouvernementales locales et nationales ainsi que des fondations privées et des organisations pour la réforme sociale avaient transmis le message concernant la réforme de la santé publique à la classe ouvrière et aux immigrants dans les villes ${ }^{43}$. Comme beaucoup de ses homologues à travers le pays, le Labor Clarion faisait des reportages réguliers sur les programmes de santé publique, répandait les conseils pour la prévention de maladies comme la typhoïde et la diphtérie et rendait compte des débats agitant la profession médicale à propos de la vaccination, de la désinfection et de la quarantaine. Les reportages sur la santé mettaient en avant la nécessité d'aboutir à une hygiène personnelle et au contrôle permanent des installations sanitaires de l'environnement ${ }^{44}$. Outre le soutien aux programmes de santé publique et la diffusion de conseils sanitaires, le Clarion et les dirigeants syndicaux se retrouvèrent impliqués dans la production de la compétence sanitaire. Les dirigeants ouvriers de tout le pays organisèrent des comités sanitaires pour évaluer l'efficacité des lois sur la santé des municipalités et de l'État, proposèrent leurs compétences pour les questions d'hygiène industrielle et participèrent aux comités municipaux sur la santée ${ }^{45}$. Dans leur participation à la diffusion de conseils de santé et dans la création d'une politique de la santé, les dirigeants syndicaux mirent l'accent sur la menace que faisait courir la maladie à la vie et au cadre de vie.

L'inquiétude du monde du travail et du monde médical au sujet de l'afflux d'immigrants asiatiques et de leurs produits manufacturés aux États-Unis se transforma logiquement en une inquiétude insistante à l'égard des dangers des produits importés. Cigarier et ancien soldat, William Backner, de Springfield dans le Massachusetts, craignait les dangers des «cigares de Manille» importés par le "Tobacco Trust» dénué de scrupules. Dans une lettre ouverte à George Perkins, président de l'ICMU, Backner s'opposait à la loi du Congrès visant à réduire les tarifs douaniers avec les Philippines, colonie nouvellement acquise par les États-Unis. La lettre fut imprimée dans les publications syndicalistes à travers tout le pays y compris dans le Labor Clarion. Le texte de Backner révèle une manipulation étonnante des langages racialement codés de santé et d'hygiène et son application à n'importe quelle population « racialisée » :

Maintenant chaque soldat connaît le manque de propreté des Philippins moyens et si vous le lui demandez il vous dira que plus d'un pauvre gars est revenu dans un cercueil pour les avoir touchés de trop près car ils sont un poison pour l'homme blanc. Ils souffrent tous de maladie de peau et ils sont, pour la plupart, couverts de plaies ouvertes et de cicatrices. La lèpre, le béribéri, le choléra, la peste bubonique et autres maladies infectieuses sont, comme chacun le sait, très fréquents là-bas. Ils 
sont assis à moitié nus, ils travaillent et se grattent tandis que l'air est saturé par l'odeur du poisson pourri et de l'huile de noix de coco dont les femmes s'enduisent les cheveux. Imaginez maintenant un de ces indigènes dont les dents sont devenues noires de pourriture en raison de la mastication constante de la noix de bétel, mordant les bouts [des cigares], ce que j'ai tout particulièrement observé, et utilisant leur salive pour aider à coller les bouts sur leur ouvrage, vous aurez alors une idée de ce que le fumeur américain obtiendra lorsque le trust [des Tabacs] déversera sur le marché ces cigares de Manille de grande renommée. Le gouvernement des États-Unis dépense des milliers de dollars pour faire observer la quarantaine contre ces maladies asiatiques, et lorsqu'un asiatique quitte son île pour venir dans ce pays, lui-même et ses effets sont minutieusement désinfectés et en dépit de tout cela nos législateurs proposent de donner leur approbation à ce projet de loi qui mettra dans la bouche de milliers de nos concitoyens un des plus prolifiques moyens de contamination, et si, comme je le crois fermement, cela sert à ce que le sang des Américains soit contaminé par ces sales maladies asiatiques, l'administration actuelle pourra s'en féliciter ${ }^{46}$.

Les craintes de Backner au sujet d'une invasion étrangère d'épidémies et le fait qu'il attribue les maladies endémiques aux races asiatiques offraient une nouvelle version du "péril jaune» qui avait préoccupé les officiels de la santé publique et les dirigeants ouvriers du XIXe siècle. Ses descriptions et ses peintures des " habitudes répugnantes " des cigariers de Manille reproduisaient à la fois le détail et l'emphase narrative qui avaient été employés dans les exposés sur le travail dans les ateliers des quartiers est de New York et du Chinatown de San Francisco au XIXe siècle.

Ces schémas manifestaient beaucoup plus que la familiarité de Backner avec la rhétorique des réformateurs urbains et des médecins de la santé publique de son époque; ils prouvaient à quel point les syndicalistes avaient adapté les inquiétudes et les conclusions du comité national de vigilance sur la santé publique à leur propre volonté d'acquérir des moyens d'existence sûrs et sans danger. Cette préoccupation pour le maintien d'une bonne santé malgré la précarité et la difficulté devait avoir éveillé des résonances avec leur propre expérience vécue où la maladie et l'invalidité menaçaient fréquemment la survie économique. Le fait d'attribuer sans cesse les épidémies violentes et les maladies débilitantes aux immigrants à la fois dans les discours médicaux et dans ceux des syndicats pendant le XIXe siècle était passé dans le «bon sens » irraisonné.

Les campagnes du début du XXe siècle en faveur d'une consommation saine avaient renouvelé ce mélange infâme de danger racial, d'insidieuse catastrophe sanitaire et de travail déloyal. Leur rhétorique allait avoir un héritage durable dans le syndicalisme et la politique nationale en particulier dans l'après Seconde Guerre mondiale, lorsque les syndicats ont dénoncé le risque de déclin des ouvriers américains dans le sillage de la compétition étrangère. Bien que les ouvriers syndiqués se soient reconnus comme les sujets reconnaissants de la réforme de santé publique concernant leurs lieux de travail et les améliorations de leurs conditions de vie, ils restaient vulnérables à toute aberration du système du travail - un système caractérisé par des salaires inhumains, des conditions de travail insalubres et sans sécurité et le travail d'ouvriers privés de droits (ouvriers des « ateliers », prisonniers, « classes colonisées » et minorités raciales).

Les batailles réformistes progressistes pour s'assurer de la salubrité des usines et pour faire cesser des pratiques comme le travail des enfants furent tragiquement happées dans un tourbillon de peurs et de phobies raciales endémiques dans l'expérience américaine. $\mathrm{Au}$ lieu d'élargir le combat pour les réformes de santé publique aux populations vulnérables et privées de droits comme les minorités raciales et les nouveaux immigrants, 
les syndicats ouvriers ont maintenu une limite raciale à la définition du caractère «public » de la santé et de la protection sociale - la même limite très exactement qu'ils fixaient lorsqu'ils décrivaient l'adhérent au syndicat. Cette limite faisait obstacle aux nobles combats visionnaires en faveur de modèles universels de santé, de travail et de conditions de vie. Les applications de la réforme progressiste restreintes aux frontières nationales et raciales condamnaient les possibilités de solidarité ouvrière de même qu'elles cautionnaient la vulnérabilité des ouvriers syndiqués et de leurs familles.

La prise de conscience du mouvement ouvrier vis-à-vis des problèmes de santé avait mélangé les craintes concernant la présence et la production des Asiatiques avec les menaces toujours renouvelées contre le "progrès ». Les réformateurs, les militants ouvriers ainsi que les hommes et les femmes du syndicat se rendaient compte que la réforme de la santé publique avait fait de la vision « du modèle de vie américain » une réalité plausible pour un nombre croissant de familles ouvrières blanches. Cependant, ce prétendu modèle de vie américain était fondé sur un ordre social où la prospérité des ouvriers dépendait de l'observance d'une stricte exclusion raciale sur le lieu de travail et la pratique rigoureuse du partage sexué des rôles dans le foyer. La jouissance de ce modèle américain reposait sur le caractère sacré et la sauvegarde de la classe ouvrière blanche et sur la prospérité de sa reproduction sociale. Dans les campagnes syndicales de consommateurs l'image du foyer avait été remaniée pour rejoindre l'image de l'idéal de l'artisan du XIXe siècle : indépendance économique masculine et vie domestique pour la femme. Il fallait que chacun dans la classe ouvrière joue le bon rôle sexué à l'intérieur de la famille nucléaire pour protéger son foyer. Les hommes de la classe ouvrière étaient responsables, en tant que pères et maris de l'hygiène des produits et les femmes de la classe ouvrière, en tant que mères et épouses, consommaient des produits fabriqués sous contrôle syndical afin d'assurer la santé de la famille et la stricte viabilité économique du foyer. Sous le parapluie du mouvement ouvrier, les travailleurs - hommes et femmes pouvaient soutenir le progrès pour leurs familles et par là même pour la nation américaine, en accomplissant leurs devoirs sexués. Cependant, ce « progrès » pouvait être interrompu à tout moment. L'invasion prévue de produits de consommation contaminés n'infecterait pas seulement une famille isolée, mais aboutirait à une inversion épouvantable de la vision réformiste du " progrès » social pour la classe ouvrière, la race blanche et la nation américaine. Le "progrès » était un allié des peurs xénophobes. Le spectre des menaces raciales continuera à hanter la classe ouvrière américaine dans sa poursuite $d u$ "modèle de vie américain». Si ce modèle s'annonçait comme l'accomplissement de la promesse du "progrès ", alors cette promesse était précaire et fut toujours attaquée et sujette à revirement. Au lieu d'attirer ces travailleurs sous-payés dans le giron de leur organisation, les dirigeants syndicalistes ont tenté d'éliminer la concurrence en politisant et en contrôlant les décisions concernant la consommation. De telles campagnes nationalistes à l'intention des consommateurs influencent encore de nos jours la réaction chauvine des organisations ouvrières américaines face à la compétition économique internationale.

Traduction Rosine Feferman 


\section{BIBLIOGRAPHIE}

\section{BARON Ava}

1989 : «Questions of Gender : DeSkilling and DeMasculinization in U.S. Printing Industry 1830-1915», in Gender and History, vol. 1, n² 2.

1991 : «Gender and Labor History : Learning from the Past, Looking to the Future», in Ava Baron (ed.) Work Engendered : Toward a New History of American Labor, Ithaca, Cornell University Press.

BORIS, Eileen,

1994 : Home to Work: Motherhood and the Politics of Industrial Homework in the United States, New York, Cambridge University Press.

CHAN, Sucheng

1986 : This Bittersweet Soil : The Chinese in California Agriculture, 1860-1910, Berkeley, University of California Press.

CHOPPE

1908 : Le Label, Paris.

COOPER, Patricia A.

1987 : Once a Cigarmaker : Men, Women and Work Culture in American Cigar Factories, 1900-1919, Urbana, University of Illinois Press.

ENGLANDER, Susan

1992 : Class Conflict and Coalition in the California Women's Suffrage Movement, 1907-1912, Lewiston NY, Edwin Mellen Press.

FRANK, Dana

1991 : «Gender, Consumer Organizing and the Seattle Labor Movement, 1919-1929», in Ava Baron (ed.), Work Engendered : Toward a New History of American Labor, Ithaca, Cornell University Press.

1994 : Purchasing Power : Consumer Organizing, Gender and The Seatle Labor Movement, 1919-1929, New York, Cambridge University Press.

MCCLAN, Charles

1988 : «Of Medicine, Race and American Law: The Bubonic Plague Outbreak of 1900», Law and Social Inquiry, vol. 13, $\mathrm{n}^{\circ} 3$.

MCCLELLAND Keith

1889 (été) : «Some Thoughts on Masculinity and the "Respectable Artisan" in Britain, 1850-1880» in Gender and History, vol. 1, $\mathrm{n}^{\circ} 2$.

MATTHEWS, Lillian Ruth

1913 : Women in Trade Unions in San Francisco, Berkeley, University of California Press.

MECKEL, Richard A. 
1990 : Save the Babies : American Public Health Reform and the Prevention of Infant Mortality, 1850-1920, Baltimore, Johns Hopkins University Press.

ROGERS, Naomi

1989 : «Germs with Legs : Flies, Disease and the New Public Health», Bulletin of the History of Medicine, vol. 63, $\mathrm{n}^{\circ} 4$.

ROEDIGER, David R.

1991 : The Wages of Whiteness : Race and the Making of the American Working Class, Londres, Verso.

SAWTELLE, Dr Mary

1878, décembre : «The Plague Spot», Medico-Literary Journal, vol. 1, n 4.

SAXTON, Alexander

1971 : The Indispensable Enemy: Labor and the Anti-Chinese Movement in California, Berkeley, University of California Press.

1990 : The Rise and the Fall of the White Republic: Class Politics and Mass Culture, Londres, Verso.

SHAH, Nayan

1995 : San Francisco's « Chinatown »: Race and and the Cultural Politics of Public Health, 1854-1952, thèse de doctorat, University of Chicago.

SPEDDEN Ernest Radcliffe

1910, The Trade Union Label, Baltimore, Johns Hopkins University Press.

Tomes, Nancy

1990 : «The Private Side of Public Health : Sanitary Science, Domestic Hygiene and the Germ Theory, 1870-1900», Bulletin of the History of Medicine, vol. 64, $n^{\circ} 4$.

TRAUNER, Joan B.

1978 : «The Chinese as Medical Scapegoats in San Francisco, 1870-1905», California History, vol. 57.

\section{NOTES}

1. « Common Council, Board of Alderman », Daily Alta California, 22 août 1854.

2. Sawtelle 1878 : 11.

3. Trauner $1978: 70-87$; McClain $1988: 447-513$.

4. The Cigar Maker's Official Journal, octobre $1878: 1$, cité par Spedden $1910: 10-11$. The Trade Union Label est un mémoire que Spedden a écrit pour un doctorat à la Johns Hopkins University.

5. Saxton 1971.

6. Roediger 1991 ; Saxton 1990.

7. « Memorial on Chinatown » Chinatown Declared a Nuisance ! : 13 ; San Francisco Board of Health, Annual Report, 1897-19898 : 259-262.

8. « Memorial on Chinatown by an Investigating Committee of the Anti-Chinese Council, WPC » in Workingmen's Party of California, Chinatown Declared a Nuisance! 1880, San Francisco : 12-13.

9. Saxton $1971: 214-215$; Cooper $1987: 10-40$.

10. Cigar Maker's Official Journal, octobre 1880 : 7, cité dans Spedden 1910 : 14-15. 
11. «Coolie » était un terme codé racialement et faisait référence aux serviteurs venant de Chine et d'Inde et envoyés en apprentissage aux Caraibes et en Amérique latine. Les militants ouvriers blancs avaient souvent accusé les immigrants chinois qui arrivaient en Amérique du Nord de faire partie du commerce des coolies qui amenait des ouvriers en apprentissage du sud de la Chine en Amérique latine . Néanmoins, comme Sucheng Chan l'a expliqué en détail, la plupart de ces hommes qui arrivaient en Amérique du nord avait acheté leur billet à crédit et n'avaient pas de contrat de travail. Voir Sucheng Chan 1986. 12. Voir CMIU n ${ }^{\circ} 228$, « Protect Home Industry » certificate, 1887, California Historical Society, Certificate Collection. Ces transformations rendirent le label adaptable aux combats syndicalistes partout dans le pays, par exemple, pendant la grève des fabricants de cigares en 1877 à New York contre la production réalisée dans les immeubles d'habitation. Boris 1994.

13. Spedden $1910: 19-22$; Choppe $1908: 221-237$.

14. Labor Clarion (LC) vol. $1 \mathrm{n}^{\circ} 19$ (4 juillet 1902); vol. $5 \mathrm{n}^{\circ} 45$ (11 janvier 1907) ; vol. $6 \mathrm{n}^{\circ} 16$ (7 juin 1907) ; vol. 8 n $^{\circ} 5$ (19 mars 1909); vol. $9 \mathrm{n}^{\circ} 38$ (4 nov. 1910).

15. $L C$, vol $1 \mathrm{n}^{\circ} 19$ (4 juillet 1902).

16. $L C$, vol. $8 \mathrm{n}^{\circ} 5$ (19 mars 1909) ; vol. $6 \mathrm{n}^{\circ} 35$ (18 oct. 1907).

17. Bien que l'utilisation explicite par William Bonsor de l'expression « mode de vie du Blanc » ait été peu fréquente, le terme 'blanc' était implicite dans la définition de « l'Américain » dans la mesure où cette expression était continuellement mise en opposition avec la « race asiatique » de même qu'elle était définie contre les Noirs, les Latino-américains et les Asiatiques aussi bien que contre les « races d'Europe du sud et de l'est » à l'intérieur des États-Unis. Bonsor 1914 : 40.

18. La politique raciale de Samuel Gompers était compliquée. En dépit de son antagonisme envers les ouvriers chinois, Gompers dénonçait de façon explicite l'exclusion des ouvriers noirs du syndicalisme.

19. AFL 1901. Publié aussi en tant que Senate Document 137 (Washington DC., GPO, 1902). Et sous forme d'une série d'articles dans le LC. Finalement l'Asiatic Exclusion League de San Francisco publia une version abrégée : Meat vs Rice (San Francisco, 1908). Sur le contexte, voir Saxton 1971 : 270-273.

20. Discours du Sénateur James G. Blaine du 14 février 1879 au Sénat des États-Unis, cité par Samuel Gompers et Herman Gutstadt, « Meat vs Rice - Part III », in LC, vol. 7 n 19 (26 juin 1908).

21. Pour plus d'informations sur les significations et les codes de la masculinité de la classe ouvrière du XIXe siècle, voir Baron 1991 : 1-46 ; Baron 1989 : 178-199 : McClelland 1989 : 164-177.

22. Boris 1994. Pour la publicité de l'époque sur ces mouvements voir LC, vol. 3, nº 20 (8 juillet 1904). Des articles sur les ateliers et la réforme de l'hygiène industrielle étaient souvent publiées dans le $L C$

23. $L C$, vol. $3 \mathrm{n}^{\circ} 28$ (2 septembre 1904).

24. $L C$, vol. $7 \mathrm{n}^{\circ} 52$ (12 février 1909).

25. $L C$, vol. $6 \mathrm{n}^{\circ} 32$ (27 septembre 1909).

26. $L C$, vol. $6 \mathrm{n}^{\circ} 47$ (10 janvier 1908). Pour d'autres exemples de publicité voir les encarts publicitaires du LC, vol. $1 \mathrm{n}^{\circ} 25$ (15 août 1902) ; vol. 2, nº 41 (4 décembre 1903) ; vol. 11, nº 3 (1er mars 1912).

27. Encarts publicitaires du LC, vol. $2 \mathrm{n}^{\circ} 13$ (22 mai 1903); vol. 3, $\mathrm{n}^{\circ} 19$ (3 juillet 1903) ; vol. 2 n $^{\circ} 45$ (1er janvier 1904); vol. 2 n 50 (5 février 1904). 
28. $L C$, vol. $1 \mathrm{n}^{\circ} 36$ (31 octobre 1902). Pour d'autres comptes rendus et appuis au syndicat blanc des fabricants de balais, voir LC, vol. $1 \mathrm{n}^{\circ} 40$ (28 novembre 1902).

29. J'ai développé ailleurs une analyse détaillée sur les caractéristiques de l'espace de travail racialisé dans les inspections de la santé publique effectuées dans les usines et les industries de service chinoises à la fin du XIXe siècle à San Francisco. Shah 1995.

30. $L C$, vol. $3 \mathrm{n}^{\circ} 20$ (8 juillet 1904).

31. $L C$, vol. $4 \mathrm{n}^{\circ} 11$ (5 mai 1905); vol. $4 \mathrm{n}^{\circ} 22$ (21 juillet 1905); vol. $4 \mathrm{n}^{\circ} 23$ (28 juillet 1905).

32. $L C$, vol. $4 \mathrm{n}^{\circ} 11$ (5 mai 1905).

33. $L C$, vol. $4 \mathrm{n}^{\circ} 50$ (2 février 1906).

34. Frank 1991 : 273-295; Frank 1994.

35. Matthews 1913.

36. LC, vol.4 $\mathrm{n}^{\circ} 2$ (3 mars 1905); vol.5 $\mathrm{n}^{\circ} 3$ (6 mars 1906); vol. $6 \mathrm{n}^{\circ} 36$ (25 octobre 1907).

37. $L C$, vol. $4 \mathrm{n}^{\circ} 35$ (20 octobre 1905).

38. $L C$, vol. $4 \mathrm{n}^{\circ} 39$ (17 novembre 1905); vol. $7 \mathrm{n}^{\circ} 1$ (21 février 1908); vol. $7 \mathrm{n}^{\circ} 10$ (24 avril 1908); vol. $8 \mathrm{n}^{\circ} 23$ (23 juillet 1909); vol. $12 \mathrm{n}^{\circ} 12$ (2 mai 1913).

39. LC, vol. $7 \mathrm{n}^{\circ} 28$ (26 août 1908) ; vol. 7 n³6 (23 octobre 1908) ; vol. $7 \mathrm{n}^{\circ} 46$ (1er janvier 1909).

40. $L C$, vol. $6 \mathrm{n}^{\circ} 47$ (10 janvier 1908).

41. Englander 1992 .

42. $L C$, vol. $8 \mathrm{n}^{\circ} 32$ (24 septembre 1909) ; vol. $8 \mathrm{n}^{\circ} 37$ (29 octobre 1909) ; vol. $10 \mathrm{n}^{\circ} 17$ (2 juin 1911) ; vol. $11 \mathrm{n}^{\circ} 7$ (29 mars 1912) ; vol. $11 \mathrm{n}^{\circ} 9$ (12 avril 1912) ; vol. $11 \mathrm{n}^{\circ} 19$ (21 juin 1912); vol. $11 n^{\circ} 29$ (30 août 1912).

43. Rogers 1989 : 599-617 ; Tomes 1990 : 409-539; Meckel 1990.

44. $L C$, vol. $1 \mathrm{n}^{\circ} 18$ (27 juin 1902) ; vol. $6 \mathrm{n}^{\circ} 52$ (14 février 1908) ; vol. $9 \mathrm{n}^{\circ} 25$ (5 août 1910); vol. 9 n $^{\circ} 27$ (19 août 1910) ; vol. 9 n $^{\circ} 28$ (26 août 1910) ; vol. 9 n 31 (16 septembre 1910); vol. $9 \mathrm{n}^{\circ} 52$ (23 septembre 1910); vol. $9 \mathrm{n}^{\circ} 46$ (13 janvier 1911); vol. $12 \mathrm{n}^{\circ} 12$ (2 mai 1913); vol. $13 n^{\circ} 31$ (11 septembre 1914) ; vol. $12 n^{\circ} 33$ (26 septembre 1913) ; vol. $13 n^{\circ} 42$ (27 novembre 1914) ; vol. $14 \mathrm{n}^{\circ} 24$ (23 juillet 1915) ; vol. $15 \mathrm{n}^{\circ} 11$ (21 avril 1916) ; vol. $15 \mathrm{n}^{\circ} 46$ (22 décembre 1916) ; vol. $15 \mathrm{n}^{\circ} 47$ (29 décembre 1916) ; vol. 17 n³8 (25 octobre 1918); vol. $19 \mathrm{n}^{\circ} 47$ (24 décembre 1920) ; vol. $19 \mathrm{n}^{\circ} 51$ (21 janvier 1921) ; vol. $20 \mathrm{n}^{\circ} 7$ (18 mars 1921) ; vol. $20 \mathrm{n}^{\circ} 12$ (22 avril 1921) ; vol. $20 \mathrm{n}^{\circ} 20$ (17 juin 1921) ; vol. $22 \mathrm{n}^{\circ} 9$ (30 mars 1923) ; vol. $22 \mathrm{n}^{\circ} 14$ (4 mai 1923) ; vol. $23 \mathrm{n}^{\circ} 40$ (31 octobre 1924) ; vol. $24 \mathrm{n}^{\circ} 4$ (27 février 1925) ; vol. 24 $n^{\circ} 8$ (27 mars 1925); vol. $26 n^{\circ} 31$ (2 septembre 1927).

45. $L C$, vol. $11 n^{\circ} 36$ (18 octobre 1912) ; vol. 8 n 46 (31 décembre 1909) ; vol. 7 nº 6 (5 juin 1908).

46. $L C$, vol. $8 \mathrm{n}^{\circ} 31$ (17 septembre 1909) : 7 .

\section{RÉSUMÉS}

$\mathrm{Au}$ tournant du siècle, les syndicats américains tendent à présenter les immigrants chinois comme la principale menace contre les travailleurs syndiqués blancs. Par une analyse des discours sur le danger racial, la santé publique et les rôles sexués qui permettent la sécurité familiale, cet article examine la façon dont l'image de la menace sanitaire représentée par les 
Chinois a fini par être partie intégrante des campagnes en direction des consommateurs orchestrées par les syndicats américains.

At the turn of century, American labor politics represented Chinese immigrants as a principal threat to the survival of white union worker. Through an analysis of discourses of racial danger, public health, and the gender roles that ensure family security, this article examines how the imagery of the Chinese medical menace became incorporated in consumer campaigns orchestrated by US labor unions.

\section{AUTEUR}

\section{NAYAN SHAH}

Nayan SHAH est Assistant Professor à l'université d'état de New York. Il a soutenu en 1995, à l'université de Chicago, sa thèse : San Francisco's Chinatown : Race and the Cultural Politics of Public Health, 1854-1982. 